\title{
PO40
}

\section{EFFECT OF LIGHTING ON READABILITY OF COLOUR PRINTING FOR VARIOUS AGES}

\author{
Natsuki Murakami et al.
}

DOI 10.25039/x47.2020.PO40

\section{Paper accepted for the $5^{\text {th }}$ CIE Symposium on Colour and Visual Appearance}

The paper was selected by the International Scientific Committee (ISC) for presentation at the 5th CIE Symposium on Colour and Visual Appearance, Hong Kong, CN, April 21-22, 2020, which, due to the corona pandemic, could not take place. The paper has not been peer-reviewed by CIE.

\section{(C) CIE 2020}

All rights reserved. Unless otherwise specified, no part of this publication may be reproduced or utilized in any form or by any means, electronic or mechanical, including photocopying and microfilm, without permission in writing from CIE Central Bureau at the address below. Any mention of organizations or products does not imply endorsement by the CIE.

This paper is made available open access for individual use. However, in all other cases all rights are reserved unless explicit permission is sought from and given by the CIE.

CIE Central Bureau

Babenbergerstrasse 9

A-1010 Vienna

Austria

Tel.: +4317143187

e-mail: ciecb@cie.co.at

www.cie.co.at 


\title{
PO40 \\ EFFECT OF LIGHTING ON READABILITY OF COLOUR PRINTING FOR VARIOUS AGES
}

\author{
Murakami, N. ${ }^{1}$, Iwata, T. $^{2}$, Sakuma, R. ${ }^{1}$, Nishihara, K. ${ }^{3}$ \\ ${ }^{1}$ Graduate school engineering of Tokai University, Kanagawa, JAPAN \\ 2 Tokai University, Kanagawa, JAPAN \\ ${ }^{3}$ Misawa Reform Co., Ltd, Tokyo, JAPAN \\ nackun919@gmail.com
}

\begin{abstract}
In recent years, LED lighting has spread rapidly and has been used in various places. Also, there has been an increase in colour printing on posters, in advertisements and on packaging using various colour for alphabetic characters and background. It is a concern that elderly people with ageing eyes which cannot read the colour characters on the colour background easily under some colour lighting. A subjective experiment was conducted on the readability of colour printing lit by different CCT lamps. The colour differences calculated by CIEDE2000 formula was smaller than the colour differences calculated by CIE76 formula under all conditions. The subject experiments showed that the readability of colour printing could not be predicted by the colour difference calculated by the CIEDE2000 formula. As a result of examination using tristimulus values, it was suggested that accurate readability evaluation can be predicted by considering colour constancy.
\end{abstract}

Keywords: LED lighting, Colour printings, Readability, Colour difference, Tristimulus value

\section{Introduction}

In recent years, LED lighting has spread rapidly and has been used in various places. The common type of LED lighting is the combination of blue LEDs with yellow and red phosphors to produce white light. Recently, the type that uses red, green, blue, and white LEDs (called RGBW) is also available. Light with various colours can be emitted from this type of LED.

Also, colour printing using various colours for alphabetic characters and background, has increased on posters, in advertisements and on packaging. As people age, the lens selectively absorbs shorter-wavelength light because of accumulation of yellow pigments in the lens (Salvi et al., 2006). It is a concern that elderly people with ageing eyes which have low transmittance for shorter wavelengths cannot read the colour characters on the colour background easily under some colour lighting.

A previous study (Komori, 2019) on the readability of colour printing using a paired comparison method showed the difference in readability between ages. However, the CIE76 colour difference model showed low correlation with readability.

The purpose of our study is to develop a method which can predict readability of colour printing under different light for various ages using the latest colour difference formula and 7-point scale. In this paper, the term "readability" refers to the visibility of the target.

\section{Previous research on colour discrimination}

\subsection{Identification of colour}

The colour appearance model is based on the tristimulus values $X Y Z$, which are calculated by the spectral distribution of the light source: $I(\lambda)$, the spectral reflectance of the target colour: $\rho$ $(\lambda)$, and the colour matching functions of the observer: $x(\lambda), y(\lambda), z(\lambda)$. The tristimulus values $X Y Z$ are calculated from Equations (1) to (3).

$$
X=\int I(\lambda) \rho(\lambda) \overline{x_{a g e}}(\lambda) d \lambda
$$




$$
\begin{aligned}
& Y=\int I(\lambda) \rho(\lambda) \overline{y_{\text {age }}}(\lambda) d \lambda \\
& Z=\int I(\lambda) \rho(\lambda) \overline{Z_{\text {age }}}(\lambda) d \lambda
\end{aligned}
$$

where

$I(\lambda) \quad$ is the spectral distribution of the light source

$\rho(\lambda) \quad$ is the spectral reflectance of the target colour

$x_{\text {age }}(\lambda), y_{\text {age }}(\lambda), z_{\text {age }}(\lambda) \quad$ are the colour matching functions of the observer

\subsection{Colour difference}

The colour difference $\triangle E^{*}$ ab calculated by the CIE76 formula is the first colour difference of CIE. $\Delta E^{*}{ }_{\text {ab }}$ is calculated by equation (4) based on the coordinates of two points in the CIELAB colour space obtained by converting the coordinates of the CIEXYZ colour space.

$$
\Delta E^{*}{ }_{a b}=\sqrt{\left(L^{*}{ }_{1}-L^{*}{ }_{2}\right)^{2}+\left(\Delta a^{*}{ }_{1}-\Delta a^{*}{ }_{2}\right)^{2}+\left(\Delta b^{*}{ }_{1}-\Delta b^{*}{ }_{2}\right)^{2}}
$$

where

$$
\begin{aligned}
& L^{*}{ }_{1}, L^{*}{ }_{2} \quad \text { are the brightness of two points } \\
& a^{*}{ }_{1}, a^{*}{ }_{2}, b^{*}{ }_{1}, b^{*}{ }_{2} \quad \text { are the coordinates representing hue and chroma }
\end{aligned}
$$

However, it was pointed out that the CIE76 formula had a low correlation with the visual sense (M.R.Luo et. al, 2001), so the CIEDE2000 formula was proposed as a new colour difference formula. There are elliptical colour identification areas in the CIELAB colour space (MacAdam, 1970). However, $\Delta E^{\star}$ ab is shown as a perfect circle on the chromaticity diagram (Tanaka et. al, 2014). It is considered to be the cause of the deviation from the human colour identification range. $\Delta E_{00}$ calculated by the CIEDE2000 formula was improved by weighting the deviation of $\Delta E^{\star}{ }_{\text {ab }}$ in consideration of brightness, chroma, and hue (M.R.Luo et. al, 2001). $\Delta E_{00}$ is calculated by equation (5) based on the LCH colour space converted from the CIELAB colour space.

$$
\Delta E_{00}=\sqrt{\left(\frac{\Delta L^{\prime}}{k_{L} S_{L}}\right)^{2}+\left(\frac{\Delta C^{\prime}}{k_{C} S_{C}}\right)^{2}+\left(\frac{\Delta H^{\prime}}{k_{H} S_{H}}\right)^{2}+R_{T}\left(\frac{\Delta C^{\prime}}{k_{C} S_{C}}\right)\left(\frac{\Delta H^{\prime}}{k_{H} S_{H}}\right)}
$$

where

$\Delta L^{\prime} \quad$ is the brightness difference

$\Delta C^{\prime} \quad$ is the chroma difference

$\Delta H^{\prime} \quad$ is the hue difference

$R_{T} \quad$ is the revolution coefficient for the calculation of blue colour differences

$S_{L}, S_{C}, S_{H} \quad$ are scaling factors

$k_{\mathrm{L}}, k_{\mathrm{c}}, k_{\mathrm{H}} \quad$ are weighting parameters

\section{Methods}

\subsection{Conditions}

\subsubsection{The spectral distribution of the light source: $I(\lambda)$}

The spectral distribution of each colour temperature $(2500 \mathrm{~K}, 5000 \mathrm{~K}, 8000 \mathrm{~K})$ of the light source used in the experiment was measured by the spectral illuminance meter CL-500A (KONICAMINOLTA). Figure 1 shows the spectral distribution of the light sources used in the experiment. 


\subsubsection{The spectral reflectance of the target colour: $\rho(\lambda)$}

The spectral reflectance of the colours of the visual targets (combination of colour characters and colour background) used in the experiment were measured by spectrophotometer CM-d700 (KONICAMINOLTA). Each target was a combination of two of seven colours (colour1: yellow, colour2: red, colour3: light blue, colour4: light yellow, colour5: pink, colour6: light green, colour7: purple). Figure 2 shows the spectral reflectance of the colours of the visual targets.

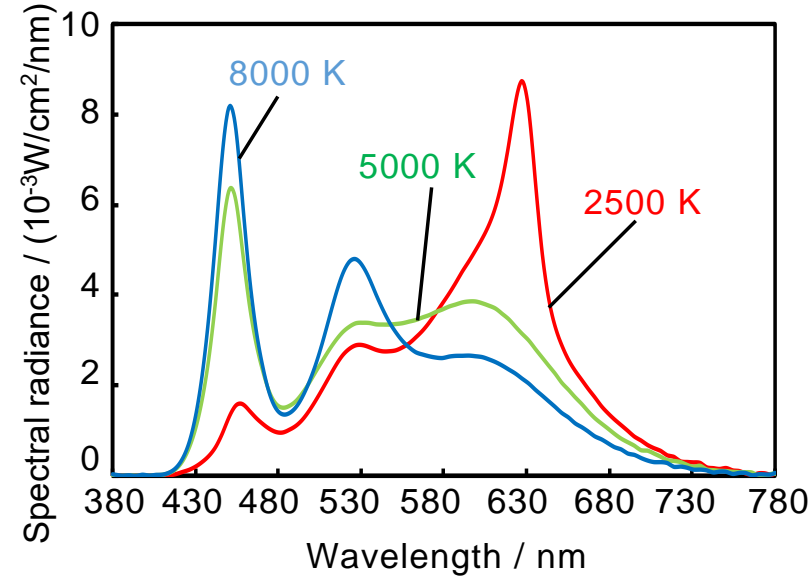

Figure 1 - Spectral distribution

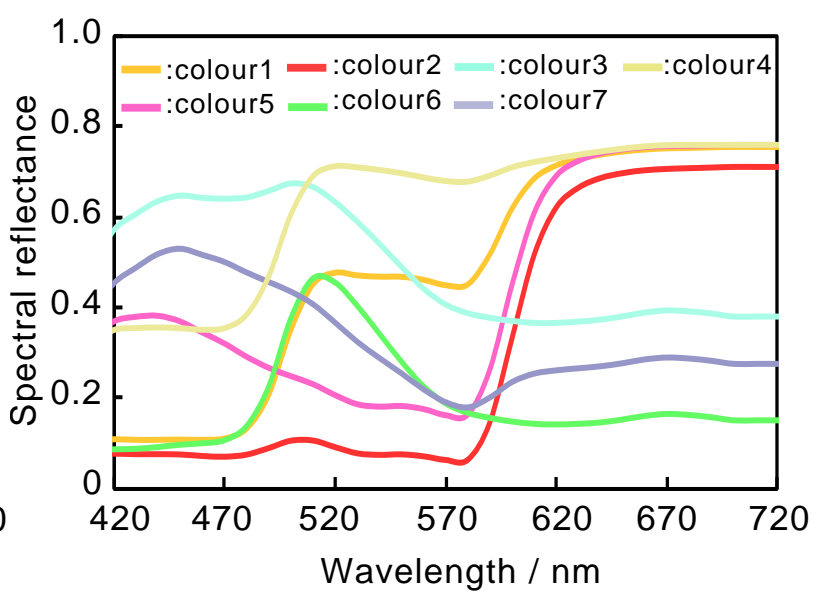

Figure 2 - Spectral reflectance

\subsubsection{The colour matching functions of observer: $x(\lambda), y(\lambda), z(\lambda)$}

The colour matching function of the person in their 20s was assumed as the CIE19312-deg function, and the colour matching function of the person in their 70 s was calculated using the visual sensitivity of a person in their 70s of JIS (JIS, 2010). Also, the colour matching functions simulating the person in their 70s were calculated from the transmittances of special goggles (Figure 3) simulating an elderly person's eye. Figure 4 shows the colour matching function of the people in their 20s, 70s, and simulated 70s.

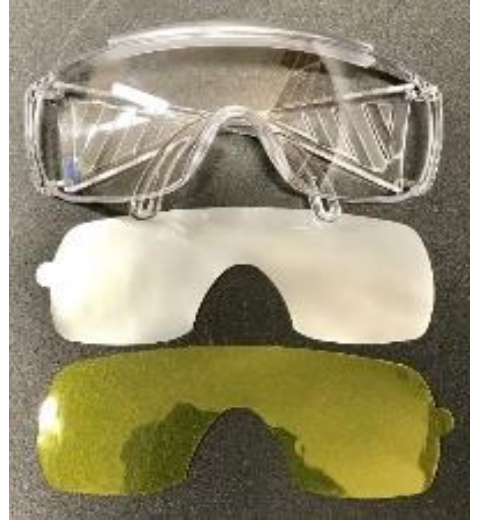

Figure 3 - Special goggle

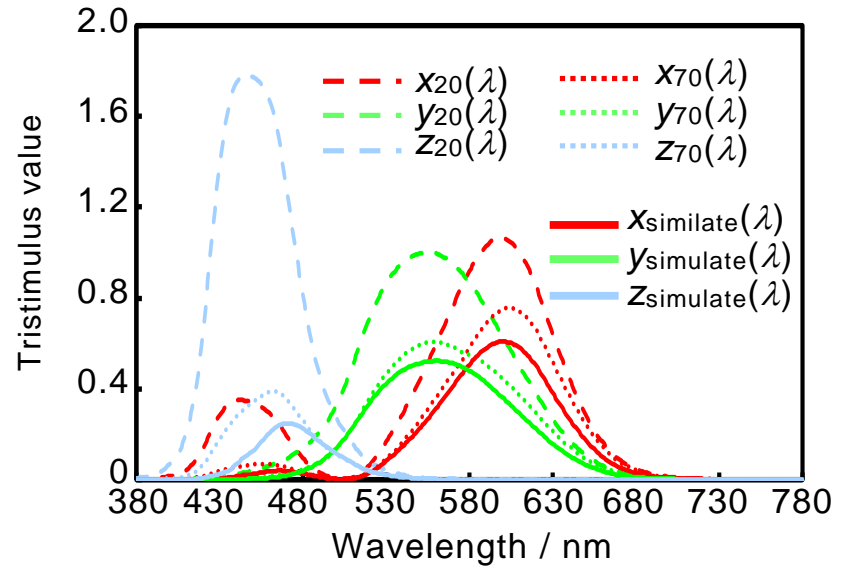

Figure 4 - Colour matching function

\subsubsection{The decision of the visual targets}

The six visual targets were selected based on the colour differences between the background and the alphabetic characters. The size of the visual targets was $100 \mathrm{~mm} \times 110 \mathrm{~mm}$, and the characters were set to 24 points so that the size was large enough to read for the elderly. Table 1 shows the colour combinations (the colour of alphabetic characters and the colour of background) of the six visual targets (A to F) shown in Figure 5. 
Table 1 - Colour combinations of six visual targets

\begin{tabular}{|c||c|c||c||c|c|}
\hline The visual target & Characters & Background & The visual target & Characters & Background \\
\hline \hline A & colour 3 & colour 2 & D & colour 5 & colour 6 \\
\hline B & olour 2 & colour 3 & E & colour 4 & colour 3 \\
\hline C & colour 6 & colour 5 & F & colour 7 & colour 1 \\
\hline
\end{tabular}

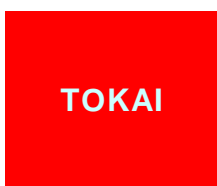

A

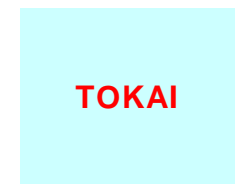

B

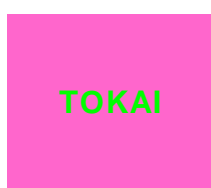

C

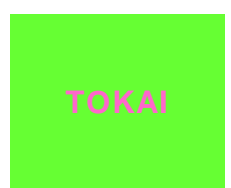

D

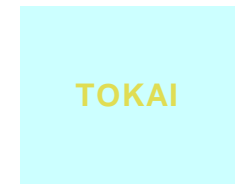

$\mathrm{E}$

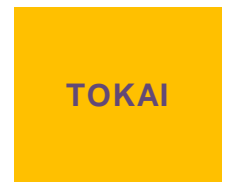

$\mathrm{F}$

Figure 5 - Six visual targets

\subsection{Experiment set-up}

An experimental booth (width $800 \times$ depth $600 \times$ height $900 \mathrm{~mm}$ ) was set up and LED lighting was installed. PHILIPS Hue with changeable colour temperature was used as the light source, and the colour temperatures were $2500 \mathrm{~K}$ (Ra 94), $5000 \mathrm{~K}$ ( $\mathrm{Ra} 94)$, and $8000 \mathrm{~K}$ (Ra 94). The illuminance on the target was kept at 500 Ix. Special goggles were used to simulate an elderly person's eye of lower transmittance of shorter wavelengths. The transmittance of the goggles at $450 \mathrm{~nm}$ was $24.3 \%$ while that at $550 \mathrm{~nm}$ was $55.4 \%$. Figure 3 shows the special goggles used in the experiment. The subjective experiment was conducted under six conditions (three colours of light with and without goggles). Figure 6 shows subjects during the experiment.

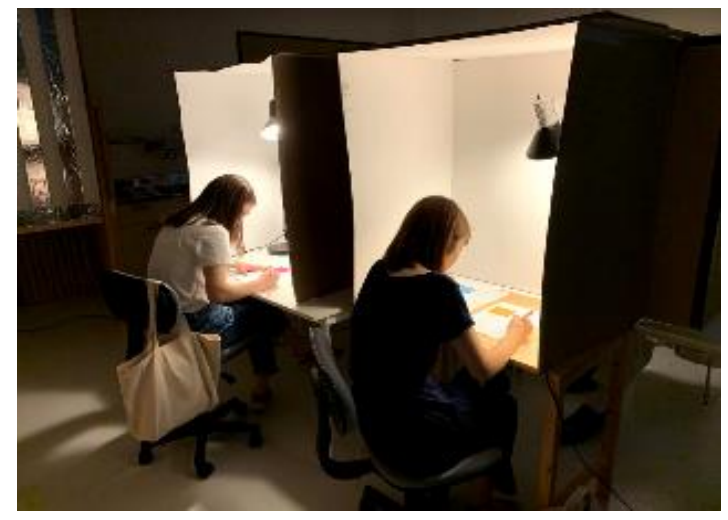

Figure 6 - Subjects during the experiment

\subsection{Procedure}

Eighteen Japanese students (between 21 and 22 years old) participated as subjects. In order to exclude colour blind subjects, Ishihara-style colour blindness tests (Ishihara, 2014) were conducted. Figure 7 shows the Ishihara-style colour blindness test. As a result of the Ishiharastyle colour blindness tests, all subjects had normal colour vision. The subjects acclimated themselves to darkness for a few minutes then looked at the visual target in the booth and assessed the readability using a 7-point scale (-3: very difficult to read, -2 : difficult to read, -1 : slightly difficult to read, 0 : neither, 1 : slightly easy to read, 2 : easy to read, 3 : very easy to read). The readability of 6 visual targets was evaluated under six conditions (three colours of light with and without goggles). 

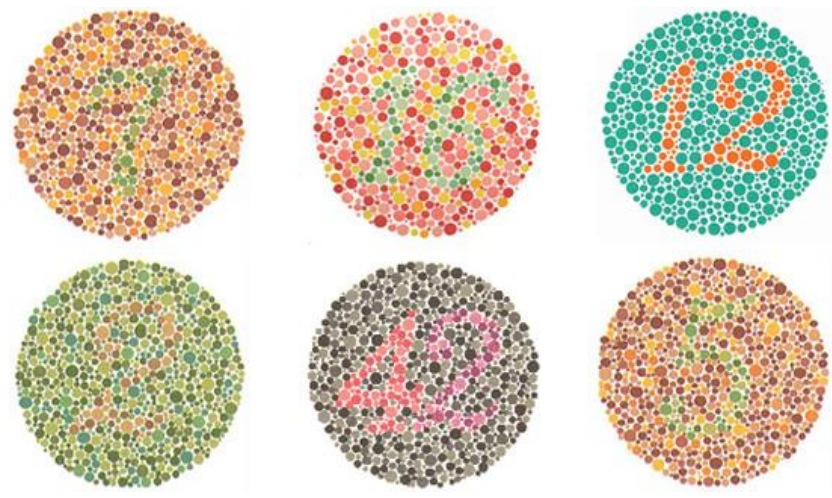

Figure 7 - Ishihara-style colour blindness test

\section{Result}

\subsection{Comparison of the CIE76 formula and the CIEDE2000 formula}

Under all conditions, $\Delta E_{00}$ calculated by the CIEDE2000 formula was smaller than $\Delta E^{*}$ ab calculated by the CIE76 formula. Figure 8 shows the relationship between $\Delta E^{*}{ }_{\text {ab }}$ and $\Delta E_{00}$ under all conditions.

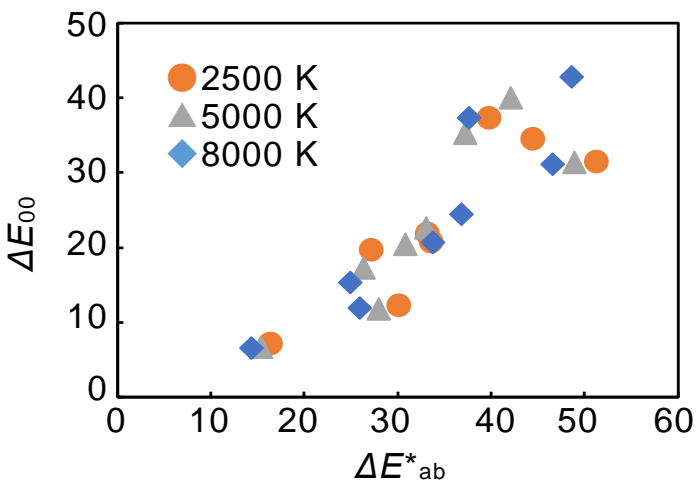

Figure 8 -Relationship between $\Delta E^{\star}$ ab and $\Delta E_{00}$

The effect of colour temperature on the colour difference $\left(\Delta E_{00}\right.$ and $\left.\Delta E^{*}{ }_{a b}\right)$ was examined and it was different for each target. In some conditions, $\Delta E_{00}$ and $\Delta E^{*}{ }_{a b}$ showed a similar tendency, e.g. both $\Delta E_{00}$ and $\Delta E^{*}{ }_{a b}$ of the visual target $E$ decreased as colour temperature increased with and without goggles, as shown in the left graph of Figure 9 . On the other hand, $\Delta E_{00}$ and $\Delta E^{*}{ }_{a b}$ are affected differently by colour temperature, as shown in the right graph of Figure 9.
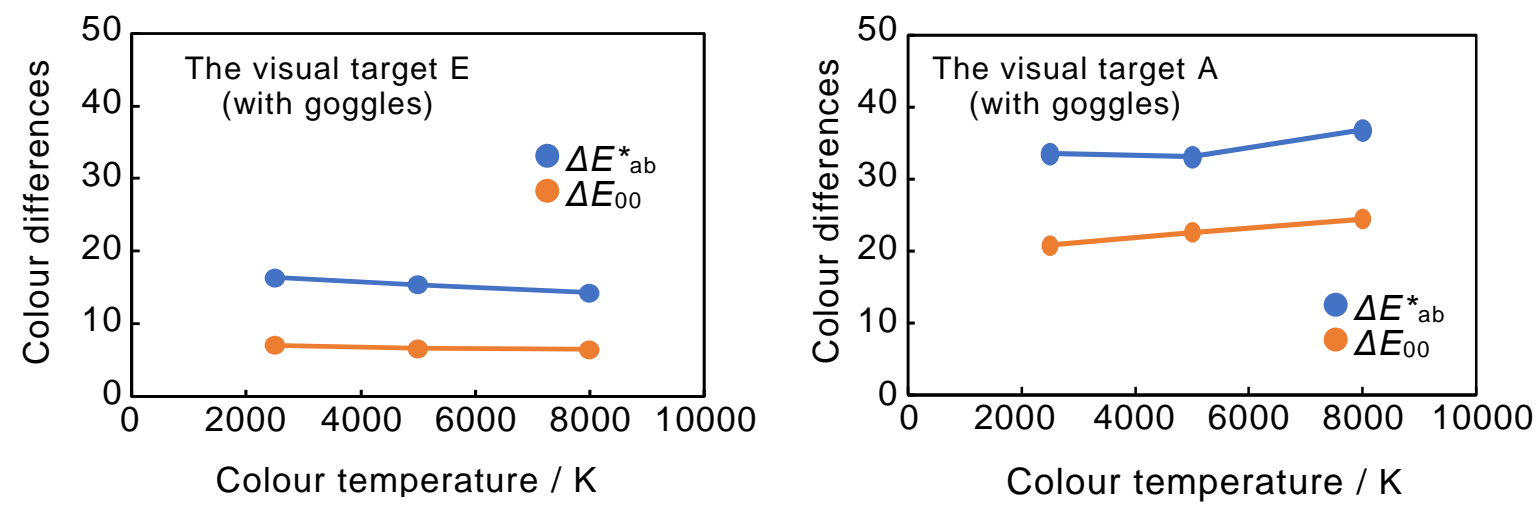

Figure 9 - Examples of the effect of colour temperature on colour difference 


\subsection{Colour difference and readability evaluation}

Figure 10 shows colour difference $\Delta E_{00}$ and the evaluation of readability. When the goggles were not worn, zero and more of the evaluation of readability was obtained under most visual targets and conditions. In addition, the correlation between the colour difference $\Delta E_{00}$ and the evaluation of the readability was low with and without the goggles. In some conditions, the readability evaluation was significantly different even when the difference in $\Delta E_{00}$ was small. Since the colour difference $\Delta E_{00}$ is an index that mainly distinguishes two very similar colours so it is considered that $\Delta E_{00}$ cannot predict the readability of colour characters on a colour background.

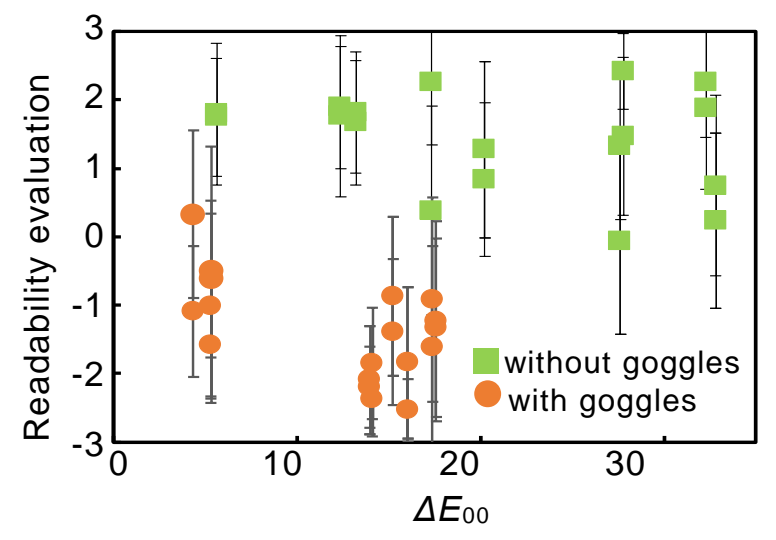

Figure 10 - Colour difference and readability evaluation

\subsection{Factors determining readability}

\subsubsection{Tristimulus value difference}

Since the evaluation of readability could not be predicted by the colour difference $\Delta E_{00}$, this paper tried to predict the evaluation of readability using the difference between the tristimulus values of the colour of the characters and the colour of the background $(\Delta X, \Delta Y$, and $\Delta Z$ ). Multiple regression analysis was performed with the differences of tristimulus values $\Delta X, \Delta Y$, and $\Delta Z$ as the explanatory variables and the evaluation of readability as the objective variable. Multicollinearity was excluded. Table 2 shows the results of the multiple regression analysis. Although the multiple correlation coefficient was 0.76 , the difference between the predicted value of the readability and the actual evaluation of the readability was large when the readability evaluation was high. Figure 11 shows the relationship between the readability evaluation and the predicted evaluation.

Table 2 - Multiple regression analysis of readability evaluation and $\Delta X, \Delta Y$, and $\Delta Z$

\begin{tabular}{|c||c|c|c|}
\hline Variable & $\begin{array}{c}\text { Partial regression } \\
\text { coefficient }\end{array}$ & $\begin{array}{c}\text { Standard partial } \\
\text { regression coefficient }\end{array}$ & Significant \\
\hline$\Delta X$ & 11.71 & 0.24 & $*$ \\
\hline$\Delta Y$ & 15.97 & 0.24 & \\
\hline$\Delta Z$ & 17.69 & 0.59 & $* *$ \\
\hline Constant term & -1.78 & & ${ }^{* *}$ \\
\hline \multicolumn{3}{|r}{${ }^{* *}: \mathrm{P}<0.01{ }^{*}: \mathrm{P}<0.05$} \\
\hline
\end{tabular}




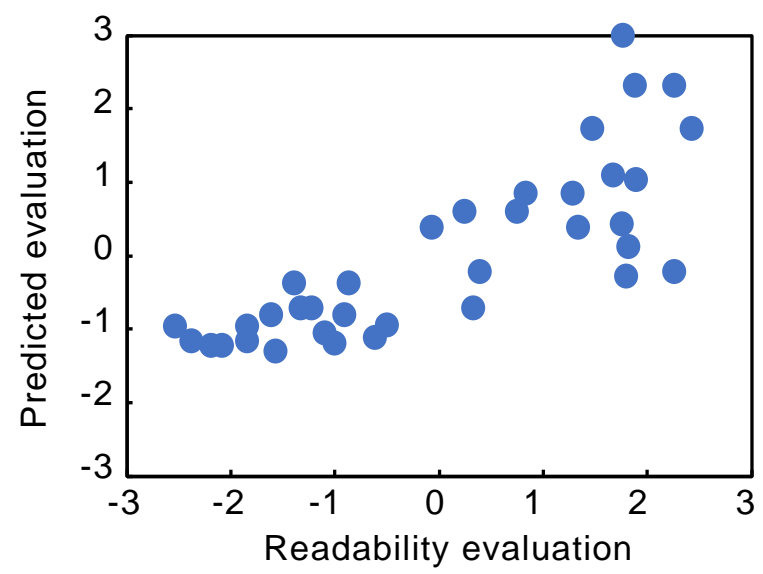

Figure 11 - Readability evaluation and prediction evaluation

\subsubsection{Consideration of other factors that affect readability}

Since the readability could not be predicted by the difference in tristimulus values, it was considered that the other factors had an effect on the readability. Three other factors, (adaptation to the colour of the background, the difference in individual sensitivity, the colour constancy was examined.

\section{(1) Adaptation to the colour of background}

Since the two visual targets where the colour combination is the same, but the background colour and the alphabetic character colour are opposite showed different readability, the effect of adapting to the background colour was examined. Multiple regression analysis was performed with the differences of tristimulus values divided by the tristimulus value of the colour of the background ( $\Delta X / X_{\text {background, }} \Delta Y / Y_{\text {background, }}$, and $\Delta Z / Z_{\text {background }}$ ) as the explanatory variable and the readability evaluation as the objective variable. The multiple correlation coefficient was 0.24 , and no effect of the colour of the background was shown. Table 3 shows results of multiple regression analysis of the readability evaluation and $\Delta X / X$ background, $\Delta Y / Y_{\text {background, and }}$ $\Delta Z / Z_{\text {background. }}$

Table 3 - Multiple regression analysis of readability evaluation and $\Delta X / X_{\text {background, }} \Delta Y / Y_{\text {background, }}$ and $\Delta \mathbf{Z} / \mathbf{Z}_{\text {background }}$

\begin{tabular}{|c||c|c|c|}
\hline Variable & $\begin{array}{c}\text { Partial regression } \\
\text { coefficient }\end{array}$ & $\begin{array}{c}\text { Standard partial } \\
\text { regression coefficient }\end{array}$ & Significant \\
\hline \hline$\Delta X / X_{\text {backgraound }}$ & -1.16 & -0.25 & \\
\hline$\Delta Y / Y_{\text {backgraound }}$ & -0.03 & -0.01 & \\
\hline$\Delta Z / Z_{\text {backgraound }}$ & 0.71 & 0.30 & \\
\hline
\end{tabular}

\section{(2) Difference in individual sensitivity}

Assuming that there is individual difference in the sensitivity of $X, Y$, and $Z$, the correlation coefficient between the differences in $X, Y$, and $Z(\Delta X, \Delta Y$ and $\Delta Z)$ and the readability evaluation was calculated for each subject. Table 4 shows the results. For all subjects the correlation coefficient between $\Delta Y$ and the readability evaluation was higher than that between $\Delta X$ and the readability or between $\Delta Z$ and the readability. However, the correlation coefficients between $\Delta Y$ and the readability evaluation were low. The individual difference in the $X, Y$ and $Z$ sensitivity could not explain the readability evaluation obtained in this paper. 
Table 4 - Correlation coefficient between the difference in the tristimulus values and the readability evaluation

\begin{tabular}{|c||c|r|r||c||c|c|c|}
\hline \multicolumn{1}{|c||}{} & \multicolumn{3}{c||}{$\begin{array}{c}\text { Correlation coefficient } \\
\text { with readability evaluation }\end{array}$} & & \multicolumn{3}{c|}{$\begin{array}{c}\text { Correlation coefficient } \\
\text { with readability evaluation }\end{array}$} \\
\hline \hline Subject & \multicolumn{1}{|c|}{$\Delta X$} & \multicolumn{1}{|c|}{$\Delta Y$} & \multicolumn{1}{c|}{$\Delta \mathrm{V}$} & \multicolumn{1}{c|}{ Subject } & $\Delta X$ & $\Delta Y$ & $\Delta Z$ \\
\hline \hline 1 & -0.04 & 0.18 & -0.13 & 10 & -0.18 & 0.25 & -0.03 \\
\hline 2 & -0.16 & 0.43 & 0.13 & 11 & -0.04 & 0.30 & -0.02 \\
\hline 3 & -0.14 & 0.35 & -0.05 & 12 & -0.20 & 0.25 & -0.09 \\
\hline 4 & -0.03 & 0.39 & 0.00 & 13 & -0.05 & 0.20 & -0.13 \\
\hline 5 & -0.30 & 0.19 & 0.04 & 14 & -0.11 & 0.20 & -0.19 \\
\hline 6 & -0.06 & 0.09 & -0.16 & 15 & -0.12 & 0.38 & -0.03 \\
\hline 7 & -0.34 & 0.29 & 0.09 & 16 & -0.13 & 0.24 & -0.12 \\
\hline 8 & -0.20 & 0.40 & -0.02 & 17 & -0.18 & 0.41 & -0.06 \\
\hline 9 & -0.24 & 0.18 & 0.17 & & & & \\
\hline
\end{tabular}

\section{(3) Colour constancy}

Assuming that there were influence of colour constancy, multiple regression analysis was performed for each light colour with the difference in tristimulus values as the explanatory variables and readability as the objective variable. Table 5 shows the results of multiple regression analysis of the readability evaluation and $\Delta X, \Delta Y$, and $\Delta Z$ for each colour temperature. The multiple correlation coefficients were 0.87 at $2500 \mathrm{~K}, 0.83$ at $5000 \mathrm{~K}$, and 0.93 at $8000 \mathrm{~K}$.

Table 5 - Multiple regression analysis of readability evaluation and $\Delta X, \Delta Y$, and $\Delta Z$ for each colour temperature

\begin{tabular}{|c|c||c|c|c|}
\hline $\begin{array}{c}\text { Colour } \\
\text { temperature }\end{array}$ & Variable & $\begin{array}{c}\text { Partial regression } \\
\text { coefficient }\end{array}$ & $\begin{array}{c}\text { Standard partial regression } \\
\text { coefficient }\end{array}$ & Significant \\
\hline \hline \multirow{2}{*}{$2500 \mathrm{~K}$} & $\Delta X$ & 15.04 & 0.44 & ${ }^{*}$ \\
\cline { 2 - 5 } & $\Delta Z$ & 101.50 & 0.82 & ${ }^{* *}$ \\
\cline { 2 - 5 } & Constant term & -2.14 & ${ }^{* *}$ \\
\hline \multirow{2}{*}{$5000 \mathrm{~K}$} & $\Delta Z$ & 21.50 & 0.83 & ${ }^{* *}$ \\
\cline { 2 - 5 } & Constant term & -1.24 & ${ }^{* *}$ \\
\hline \multirow{2}{*}{$8000 \mathrm{~K}$} & $\Delta Z$ & 30.62 & 0.94 & ${ }^{* *}$ \\
\cline { 2 - 5 } & Constant term & -1.74 & \multicolumn{2}{|c}{${ }^{* *}: \mathrm{P}<0.01{ }^{*}: \mathrm{P}<0.05$} \\
\hline
\end{tabular}

Figure 12 shows the relationship between milled (the reciprocal of the colour temperature) and standard partial regression coefficient. A high correlation between the milled and the standard partial regression coefficient was shown in $\Delta X(R=0.99)$ and $\Delta Z(R=1)$. Since $Z$ is based on a $S$-cone that is sensitive to the shorter wavelength light, $Z$ has a large effect when the colour temperature is high. 


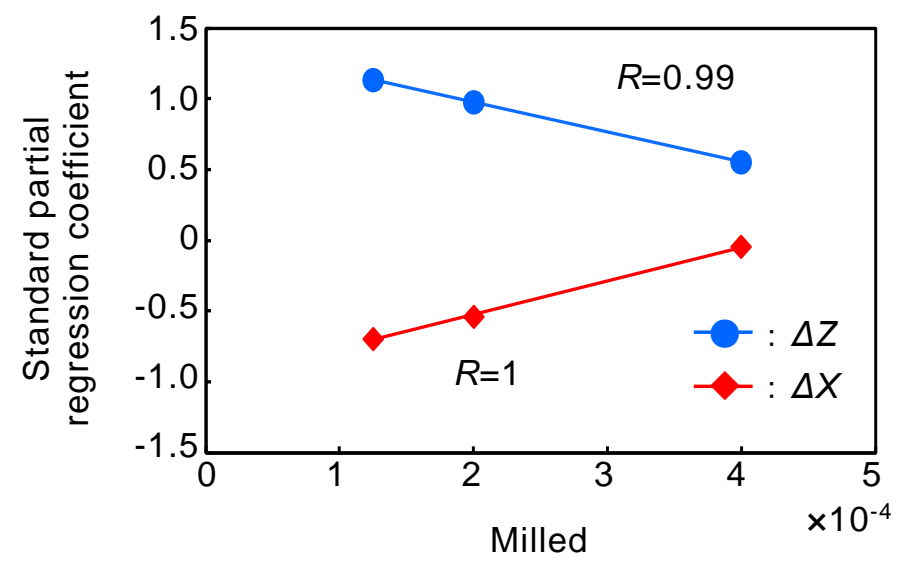

Figure 12 - Correlation between milled and standard partial regression coefficient

From the above results, to take into account colour constancy, each difference in the tristimulus values between the colour of alphabetic characters and that of the background were divided by the tristimulus values of light $X_{n}, Y_{n}$ and $Z_{n}$. A multiple regression analysis was performed with $\Delta X / X_{\mathrm{n}} \Delta Y / Y_{\mathrm{n}}$ and $\Delta Z / Z_{n}$ as the explanatory variables and the readability evaluation as the objective variable. The multiple correlation coefficient was 0.62 . It was found that colour constancy affected the relationship between the difference between the tristimulus values of the background and the alphabetic characters and the readability evaluation, however, it was not shown by the formula. A Further study is required to develop an index that can predict readability under various conditions.

\section{Conclusion}

The readability of colour printings was examined using the colour of the light source, the colour of printings, and age as parameters. The colour difference was recalculated with the CIEDE2000 formula and compared with the CIE76 formula. As a result, the colour differences calculated by the CIEDE2000 formula $\left(\Delta E_{00}\right)$ was smaller than the colour differences calculated by the $\operatorname{CIE76}$ formula $\left(\Delta E^{*}{ }_{a b}\right)$ under all conditions. However, the readability could not be predicted with the colour difference calculated by the CIEDE2000 formula $\left(\Delta E_{00}\right)$. In the examination using the difference between the tristimulus values of the colour of the characters and the colour of the background $(\Delta X, \Delta Y$, and $\Delta Z)$, it was suggested that it is possible to accurately predict the readability evaluation by considering colour constancy. However, there are still some issues to be clarified, such as the difference in the evaluation of the visual target in which the colour of the characters and the colour of the background are reversed.

\section{References}

Ishihara, S. 2014. Ishihara-style colour blindness tests 2 International version 38 figures. Tokyo: Handaya store Co., Ltd.

JIS 2010 JIS S0031:2010. Ergonomics -Accessible design- Specification of age-related luminance contrast for coloured light.

Komori, M, 2018. Effect of Lighting on colour-letter readability of various age. Tokyo Branch of The Illuminating Engineering Institute of Japan.

Luo, R, M. et. al, 2001. The Development of the CIE 2000 Colour-Difference Formula. CIEDE2000, Colour Research and Application., 26(5), 340-350.

D, L, MacAdam. 1971. Geodesic Chromaticity Diagram Based on Variances of Colour Matching by 14 Normal Observers. Applied Optics., 10(1), 1-7

Salvi, M, S. et. al, 2006. Ageing changes in the eye. Postgraduate Medical Journal., 82(971), $581-587$ 
Tanaka, K. Et. al, 2014. Study of Acceptable Colour - Difference Formula of Printed Documents. Journal of the Imaging Society of Japan., 53(2), 112-117 